\title{
Mutation frequency of Familial Mediterranean Fever and evidence for a high carrier rate in the Turkish population
}

\author{
Engin Yilmaz $^{\star 1}$, Seza Ozen ${ }^{2}$, Banu Balc1 ${ }^{1}$, Ali Duzova ${ }^{2}$, Rezan Topaloglu ${ }^{2}$, Nesrin Besbas ${ }^{2}$, \\ Umit Saatci ${ }^{3}$, Aysin Bakkaloglu ${ }^{2}$ and Meral Ozguc ${ }^{1,4}$
}

${ }^{1}$ Hacettepe University, Faculty of Medicine, Department of Medical Biology, Ankara, Turkey; ${ }^{2}$ Hacettepe University, Faculty of Medicine, Department of Pediatric Rheumatology and Nephrology, Ankara, Turkey; ${ }^{3}$ Baskent University, Department of Pediatrics, Ankara, Turkey; ${ }^{4}$ Hacettepe University, Institute of Child Health, TUBITAK, DNA-Cell Bank and Research Laboratories, Ankara, Turkey

Familial Mediterranean Fever (FMF) is a recessive disorder characterised by episodes of fever and neutrophilmediated serozal inflammation. The FMF gene (MEFV) was recently identified and four common mutations characterised. The aim of this study was to determine the carrier rate in the Turkish population and the mutation frequency in the clinically diagnosed FMF patients. We found a high frequency of carriers in the healthy Turkish population (20\%). The distribution of the five most common MEFV mutations among healthy individuals (M694V 3\%, M680I 5\%, V726A 2\%, M694I 0\% and E148Q 12\%) was significantly different $(P<0.005)$ from that found in patients (M694V 51.55\%, M680I 9.22\%, V726A 2.88\%, M694I 0.44\% and E148Q 3.55\%). European Journal of Human Genetics (2001) 9, 553-555.

Keywords: FMF; MEFV gene; mutations

\section{Introduction}

Familial Mediterranean Fever (FMF) is an autosomal recessive genetic disorder characterised by recurrent febrile episodes of fever and serosal inflammation manifested by sterile peritonitis, pleuritis and synovitis. The most serious complication of FMF is the development of amyloidosis. Colchicine has been shown to be effective in preventing the attacks of FMF as well as the development of amyloidosis. ${ }^{1}$ The disease affects populations of Mediterranean origin: Ashkenazi Jews, North African Jews, Armenians, Arabs, Italians and Turks. The gene causing FMF has been cloned in $1997 .^{2}$ The MEFV gene encodes a protein of 781 amino acids named Pyrin/Marenostrin. The $M E F V$ transcript is expressed in granulocytes that play an essential role in the inflammatory response. Four common missense mutations were identified in exon 10

*Correspondence: E Yilmaz, Hacettepe University, Faculty of Medicine, Department of Medical Biology, 06100, Sihhiye, Ankara, Turkey. Tel: +90 31230525 41; Fax: +90 31230960 60;

E-mail: engin@gen.hun.edu.tr

Received 27 November 2000; revised 28 February 2001; accepted 1 May 2001
(M694V, M694I, V726A, and M680I). Additional less common mutations have been found subsequently in exons $2,3,5,9$ and 10 bringing the current total of known mutations to 23 . $^{3}$

Recently molecular genetic studies showed that the FMF carrier frequency in the US Ashkenazi Jewish is approximately 21\%. ${ }^{4}$ Another populations survey by Stoffman et al in Israel indicated a carrier frequency of $22 \%$ in North African Jews, 39\% in Iraqi Jews, 21\% in Ashkenazi Jews and 6\% in Iranian Jews. ${ }^{5}$ A recent epidemiological study performed by Ozen et al has shown that prevalence of FMF is $1: 1075$ in the Turkish population. ${ }^{6}$

We have undertaken this study to determine the carrier frequency in the Turkish population and the mutation frequency in the clinically diagnosed FMF patients.

\section{Subjects and methods}

Routine molecular diagnosis of FMF in our laboratory began in 1999. Currently there are 550 patients in the registry and 450 of them were included in this study. The diagnosis of FMF in these patients was made according to established clinical criteria. $^{1}$ 
Carrier frequency in the population was estimated from screening samples $(n=100)$ of parents, whom were known not to have any consanguinity, of children who applied to various clinics at our Institute under consent.

Our strategy for mutation analysis includes two steps. The hot spot exon 10, which harbours 14 mutations, is first analysed by denaturing gradient gel electrophoresis (DGGE). According to the band pattern, subsequent analysis is done either by restriction endonuclease enzyme digestion or sequencing. Furthermore E148Q in exon 2 is analysed by restriction endonuclease enzyme (BstNI) digestion.

All computations were made with the use of the SPSS statistical program. The comparison of the distribution of the $M E F V$ gene mutations between FMF patients and FMF carriers was done by the $\chi^{2}$ test.

\section{Results}

Mutations in both alleles were identified in 263 of the 450 patients (58.44\%), $179(39.77 \%)$ were homozygous for the same mutation, $84(18.66 \%)$ were compound heterozygous for different combinations of the mutations. Eighty-three (18.44\%) were found to be heterozygous for one mutation. In 104 patients $(23.11 \%)$ no mutation could be detected. Mutation analysis showed that five identified missense mutations accounted for $67.64 \%$ of the 900 independent FMF alleles. The most common mutation was $\mathrm{M} 694 \mathrm{~V}$ (51.55\%). Other mutations accounted for further $16.09 \%$ of the alleles; M680I (9.22\%), E148Q (3.55\%), V726A (2.88\%) and M694I (0.44\%) (Table 1). Fourteen different genotypes were characterised in our FMF patients.

The clinical characteristics of the patients were the following: arthritis was observed in $75.18 \%$, chest pain in

Table 1 Frequency of the five common MEFV gene mutations in 450 Turkish FMF patients

\begin{tabular}{lll}
\hline Mutation & $\begin{array}{l}\text { Number of } \\
\text { alleles }\end{array}$ & $\begin{array}{l}\text { Frequency } \\
(\%)\end{array}$ \\
\hline M694V & 464 & 51.55 \\
M680I & 84 & 9.22 \\
E148Q & 29 & 3.55 \\
V726A & 28 & 2.88 \\
M694I & 4 & 0.44 \\
Unknown & 291 & 32.33 \\
\hline
\end{tabular}

$n=900$.
$37.95 \%$, peritonitis in $91.97 \%$ and vasculitic rash in $11.67 \%$. Age of onset of the patients was between 1-12 age. Almost all the patients had fever.

Sixteen patients had a history of amyloidosis (3.55\%). Among the cases with amyloidosis, 12 of them were homozygous for M694V mutation, one of them was compound heterozygous for M694V and M680I mutations and one of them had a complex allele for E148Q/E148QM694V mutations. No mutation could be detected in two of them.

Carrier frequency in the general population was done with screening for the most common mutations (Table 2). We identified one adult who was compound heterozygous for V726A and E148Q mutations (age 38). Her parents inherited separately these two mutations. She had no signs or symptoms of FMF attacks or amyloidosis.

Frequencies of E148Q, M680I, M694V, and V726A mutations in the Turkish general population were $0.06(12 / 200)$ (95\% CI $=0.06-0.15), 0.025$ (95\% CI=0.003-0.04), 0.015 (95\% CI=0.002-0.03), and $0.010(95 \% \mathrm{CI}=0.003-0.02)$.

\section{Discussion}

In this study, we have determined the mutation frequencies of five common mutations in the $M E F V$ gene in $450 \mathrm{FMF}$ patients and established the carrier frequency of the Turkish population. The M694V mutation, which is found in $40-$ $90 \%$ of different populations, ${ }^{7}$ was found in about $51.55 \%$ of Turkish FMF alleles. The M680I mutation, which is known to be common in Armenians, was found in 9.22\% of our FMF alleles. Other mutations accounted for further $6.87 \%$ of the alleles, (V726A 2.88\%, M694I 0.44\% and E148Q 3.55\%). Two preliminary studies from Turkey have shown approximately the same frequency for the M694V mutation. ${ }^{8,9}$ In one study the frequency of M680I, M694I and V726A mutations was much higher when compared to our results. Further studies analysing geographic areas are needed for final conclusions.

At the end of our study we have 291 unidentified alleles. A proportion of these alleles will be carrying rare mutations in the unscreened exons. Furthermore, regulatory region mutation and mutation in the untranslated region of the gene is still at large. Furthermore, in a group of families, the disease may not be linked to the known chromosome locus (16p13.3) as indicated by a previous study. ${ }^{10}$

Most previous studies trying to establish a genotypephenotype correlation could not show a clear relation.

Table 2 Frequency of the five common MEFV gene mutations in 100 healthy individuals

\begin{tabular}{lllllll}
\hline M694V & M680I & V726A & M694I & E148Q & Mutation frequency & Carrier rate \\
\hline $3 / 200$ & $5 / 200$ & $2 / 200$ & $0 / 200$ & $12 / 200$ & $22 / 200$ & $20 / 100$ \\
$(0.002-0.03)$ & $(0.003-0.04)$ & $(0.003-0.02)$ & $(0-0.02)$ & $(0.02-0.09)$ & $(0.06-0.15)$ & $(0.12-0.28)$ \\
\hline
\end{tabular}

${ }^{\mathrm{a}}$ One individual was compound heterozygote for V726A/E148Q mutations. ( $\left.n=200\right)$. 
M694V mutation is probably a risk factor for amyloidosis. However, amyloidosis may and does occur in patients carrying other alleles as well. In our present study, the number of amyloidosis is low $(n=16)$ therefore a clearcut analysis at this point is not possible.

In this study we also established the frequencies of $M E F V$ gene mutations in healthy individuals of Turkish population. A previous study based on clinical criteria indicated a prevalence of FMF in $1 / 1070$ in Turks. Our study results reveal that carrier rate is $20 \%$ in the Turkish population. The results of the carrier rates in Turkish population are similar to Ashkenazi and North African Jews. ${ }^{6}$ Even though the M694V mutation was the most common mutation in the FMF patients $(51.55 \%)$, it was detected in only $3 \%$ of the general population $(P<0.005)$. In contrast $\mathrm{E} 148 \mathrm{Q}$ was very frequent $(12 \%)$ among the population, but was found in only $3.55 \%$ of the FMF patients. This suggests that, this mutation may be associated with a high non-penetrance rate. Previous reports indicated that, individuals who were homozygous or compound heterozygous for E148Q, may lack clinical features.

The high incidence of $M E F V$ gene mutations in the Turkish population (gene frequency of $0.011 ; 95 \% \mathrm{CI}=0.06-0.15$, and carrier rate of $0.20 ; 95 \% \mathrm{CI}=0.12-0.28$ ) indicate that newborn screening may be discussed in the future. Further studies are needed to define the heterozygote advantage in the region remains to be defined.

\section{References}

1 Livhen A, Langevitz P, Zemer D et al: Criteria for the diagnosis of familial Mediterranean fever. Arthritis Rheum 1997; 40: 18791885.

2 International FMF Consortium: Ancient missense mutations in a new member of the RoRet gene family are likely to cause familial Mediterranean fever. Cell 1997; 90: 797-807.

3 Touitou I: The spectrum of mutations. II International conference of familial Mediterranean fever. Antalya, Turkey, 2000, pp 3-26.

4 Aksentijevich I, Torosyan Y, Samuels J et al: Mutation and haplotype studies of familial Mediterranean fever reveal new ancestral relationships and evidence for a high carrier frequency with reduced penetrance in the Ashkenazi Jewish population. Am J Hum Genet 1999; 64: 949 - 962.

5 Stoffman N, Magal N, Shohat N et al: Higher than expected carrier rates for familial Mediterranean fever in various Jewish ethnic groups. Eur J Hum Genet 2000; 8: 307 - 310.

6 Ozen S: Vasculopathy, Behçet's syndrome, and familial Mediterranean fever. Curr Opin Rheumatol 1999; 11: 393 -398.

7 Dode C, Pecheux C, Cazeneuve C et al: Mutations in the MEFV gene in a large series of patients with a clinical diagnosis of familial Mediterranean fever. Am J Hum Genet 2000; 92: 241 246.

8 Chen X, Fischel-Ghodsian N, Cercek A et al: Assessment of pyrin gene mutations in Turks with familial Mediterranean fever (FMF). Hum Mutation 1998; 11: 456-460.

9 Yalçinkaya F, Çakar N, Misirliolu M et al: Genotype-phenotype correlation in a large group of Turkish patients with familial Mediterranean fever: evidence for mutation-independent amyloidosis. Rheumatology 2000; 39: 67-72.

10 Akarsu AN, Saatci Ü, Ozen S et al: Genetic linkage study of familial Mediterranean fever (FMF) to $16 \mathrm{p} 13.3$ and evidence for genetic heterogeneity in the Turkish population. J Med Genet 1997; 34: 573-578. 